\title{
LA INVESTIGACIÓN EN ARQUITECTURA
}

Editor: John Arango Flórez

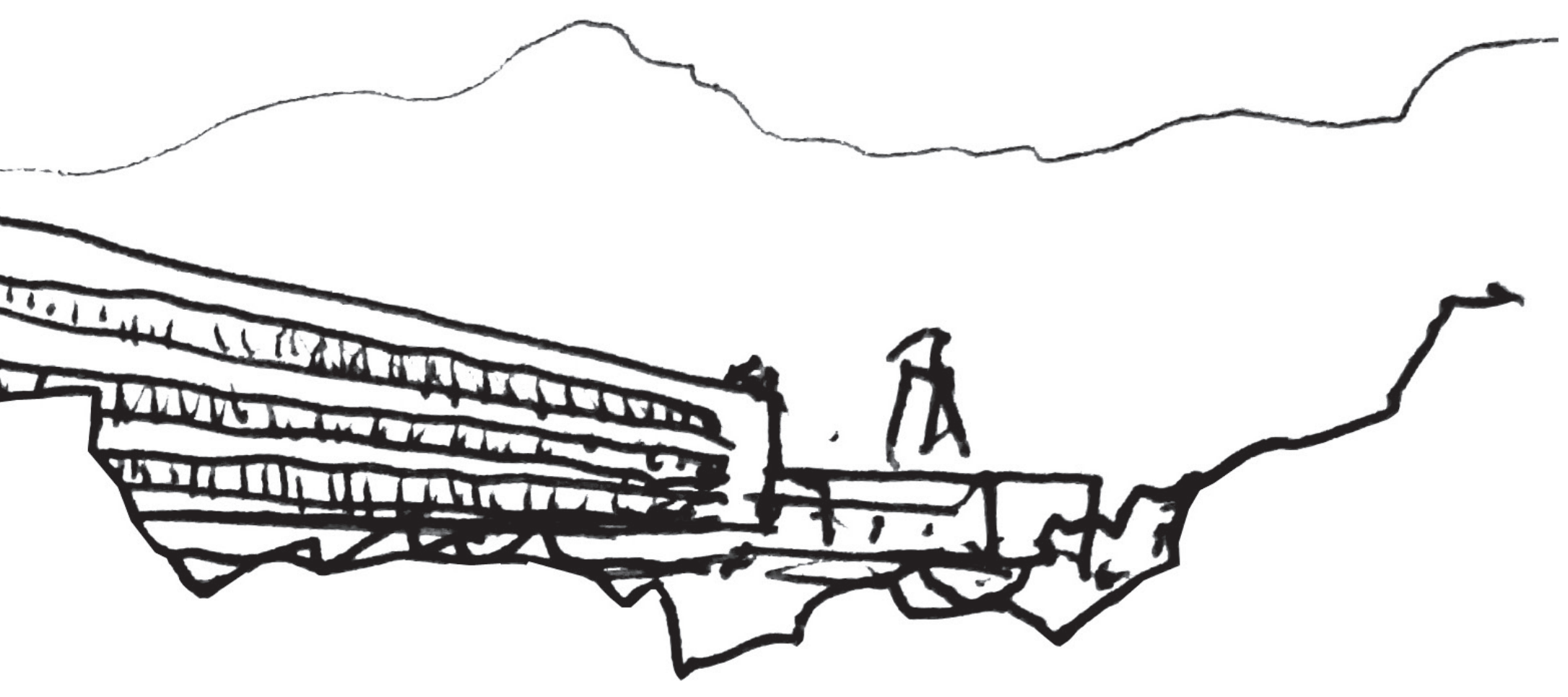






\section{La investigación en arquitectura}

Editor: John Arango Flórez 
Arango Flórez, John

La investigación en arquitectura/ John Arango Flórez (y otros diez); Editor: John Arango Flórez, Bogotá: Universidad Santo Tomás, 2017.

240 páginas; fotografías a color, ilustraciones, planos,

Incluye referencias bibliográficas

ISBN: 978-958-782-039-3

E-ISBN: 978-958-782-040-9

1. Arquitectura 2.Arquitectura - Diseño 3. Arquitectura - Medellín (Antioquia, Colombia) 4. Urbanismo - Medellín (Antioquia, Colombia) 5. Edificios, estructuras, etc. 6. Arquitectura Moderna 7. Fotografía arquitectónica 7. Investigación industrial. Universidad Santo Tomás (Colombia).

CDD 728

CO-BoUST

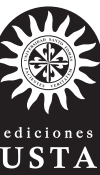

(c) John Arango Flórez, Leonardo Correa Velásquez, Lina María González Correa, Farhid Maya Ramírez, Carlos Mario Pérez Nanclares, Natalia Pérez Orrego, Juan Alberto Restrepo Sánchez, Viviana Rodríguez Ceballos, Óscar Mauricio Santana Vélez, Juan Fernando Valencia, Felipe Villa Montoya. Editor: John Arango Flórez

(c) Universidad Santo Tomás

Ediciones USTA

Carrera 9 n. ${ }^{\circ}$ 51-11

Edificio Luis J. Torres, sótano 1

Bogotá, D. C., Colombia

Teléfonos: (+571) 5878797, ext. 2991

editorial@usantotomas.edu.co

http://ediciones.usta.edu.co

Coordinación de libros: Karen Grisales Velosa

Asistencia editorial: Andrés Felipe Andrade

Diseño y diagramación: María Paula Berón

Diseño de cubierta: María Paula Berón

Corrección de estilo: Nathalie De la Cuadra N.

Universidad Santo Tomás Sede Medellín

Unidad de Investigación

Director Unidad de Investigación: Camilo Andrés Flórez Velásquez

Hecho el depósito que establece la ley

ISBN: 978-958-782-039-3

e-ISBN: 978-958-782-040-9

Impreso en Colombia • Printed in Colombia

Impreso por: Grupo Dao Digital, S.A.S.

Primera edición: 2017

Todos los derechos reservados

Se prohíbe la reproducción total o parcial de esta obra, por cualquier medio, sin

la autorización previa por escrito de los titulares. 


\section{Agradecimientos}

El editor agradece de manera especial, la confianza depositada en esta labor de curaduría académica por parte de Gabriel Obando López, decano de la Facultad de Arquitectura de la Universidad Santo Tomás, sede Medellín; quien nunca dudó de que esta compilación terminaría en el excelente producto que se presenta en estas páginas.

Gracias a Alejandro Saldarriaga y Valentina Mejía Amézquita, cuyas lecturas críticas lograron darle un norte a este libro y, por supuesto, gracias a los autores que amablemente presentaron sus conocimientos y sus trabajos investigativos de manera absolutamente desinteresada.

La edición de este libro se realizó por la iniciativa de la Unidad de Investigaciones de la Universidad Santo Tomás y a la Facultad de Arquitectura, sede de Medellín, las cuales brindaron todos los recursos intelectuales, logísticos y económicos para lograr este documento. 



\section{Contenido}

Prólogo del editor

El complejo panorama de la investigación proyectual en arquitectura y diseño

Natalia Pérez Orrego

John Arango Flórez

¿Qué es proyectar?

Investigación proyectual

Referencias

La Unidad Vecinal Modelo del plan piloto para Medellín

Una aproximación a las determinantes proyectuales

Juan Alberto Restrepo Sánchez

El plano de la Unidad Vecinal Modelo para Medellín

y su problematicidad inherente

Quiebres conceptuales y aproximaciones problemáticas 34

Intenciones en la unidad vecinal $\quad 42$

Dispositivos en la unidad vecinal $\quad 49$

Conclusiones $\quad 55$

Referencias 


\section{Habitar, TRABAJAR, cultivar cuerpo y espíritu, circular}

De la fábrica al conjunto industrial, Compañía

Colombiana de Tabaco S.A. Medellín, Colombia

Oscar Mauricio Santana Vélez

La fábrica como ciudad

La fábrica como conjunto. Edificio industrial

y conjunto industrial

La fábrica como arquitectura

Conclusiones

Referencias

La planta baja, unión de diferencias

Carlos Mario Pérez Nanclares

Introducción

Metodología

Discusión

El vacío urbano como mediador

La plaza en esquina

La plaza lateral

El carácter público en las plantas bajas

Bifurcación del plano urbano

Conclusiones 
La forma visual en la fotografía de la arquitectura moderna en Medellín (1940-1970)

Felipe Villa Montoya

Lina María González Correa

Leonardo Correa Velásquez

$\begin{array}{lc}\text { Introducción } & 123 \\ \text { Antecedentes } & 124 \\ \text { La fotografía de arquitectura } & 126 \\ \text { en Colombia } & 127 \\ \text { La fotografía de arquitectura } & 128 \\ \text { en Medellín } & 129 \\ \text { Marco teórico } & 130 \\ \text { La percepción de la forma visual en las fotografías } & 132 \\ \text { El medio de la forma visual } & 138 \\ \text { Metodología } & 144 \\ \text { Conclusiones } & \\ \text { Referencias } & \end{array}$

Construir el paisaje, la casa de vidrio de Lina Bo Bardi, 1949-1951

Farhid Maya Ramírez

Referencias 


\section{Cubierta plana en la Casa Farnsworth}

Baukunst. Arte, construcción y filosofía

Juan Fernando Valencia

$\begin{array}{lc}\text { Introducción } & 161 \\ \text { Abstracción y técnica } & 163 \\ \text { Levedad en el plano de cubierta } & 165 \\ \text { Bombilla de incalculables watts } & 167 \\ \text { Estructura de cubierta al servicio utilitario de ventilar } & 168 \\ \text { Conclusiones } & 170 \\ \text { Referencias } & 171\end{array}$

\section{El lugar interior}

La construcción de campos espaciales a partir de muebles en la casa Tugendhat de Mies van der Rohe John Arango Flórez

Introducción

Objetos como lugares

Referencias 
El papel de la mujer en el diseño de la casa moderna

El caso de la cocina como laboratorio proyectual

Viviana Rodríguez Ceballos

Introducción

187

Las ingenieras domésticas americanas

189

La ciencia de la eficacia en el hogar

191

De la monótona cocina disgregada a la cocina

compacta multifuncional

La Bauhaus y la Haus am Horn

Margarette Shütte-Lihotsky y Charlotte Perriand.

Las soluciones a la cocina moderna

Charlotte Perriand. La cocina como el contenedor de la familia

Conclusiones

205

Referencias 
Observando la piel de la ciudad

Grafiti y pintadas comerciales como artífices

de conflictos en la apropiación del espacio público

de la calle Ayacucho en Medellín

Nino Andrey Gaviria Puerta

Introducción

Marco conceptual

Marco contextual 228

Conclusiones

Referencias 


\section{Prólogo del editor}

Investigar en las disciplinas creativas como la arquitectura y el diseño plantea retos para los diseñadores-investigadores en nuestro contexto académico y cultural, y se propone, en los capítulos que vienen, como un verdadero campo de generación de conocimiento desde la práctica proyectual de la arquitectura. El libro que se presenta aquí es una muestra de los esfuerzos que la Universidad Santo Tomás, sede Medellín, y muchos proyectistas-investigadores están realizando para enriquecer el acervo epistemológico de la arquitectura y el diseño desde el entendimiento de la práctica proyectual, entendiéndola no solo como una manera de operar para darle forma al mundo a través del espacio o los objetos, sino también como la vía natural para conocer, entender y analizar la propia práctica profesional y su influencia en la cultura y en nuestro tiempo.

El esfuerzo que implicó compilar estos textos y reunirlos en un sólo volumen responde a la intención de demostrar que, en estas disciplinas que implican la creación, la investigación también hace parte fundamental del que hacer ya que basa su episteme en una metodología en sí misma investigativa, la proyectación, como se muestra en el primer capítulo que además funciona como introducción. En este se propone una especie de estado actual de la investigación proyectual y se plantean los retos y características que esta manera de ver el mundo tiene para la generación de conocimiento, es este un texto que sirve como marco para entender las diversas miradas que aparecen en el resto del libro.

Todos los textos presentados a continuación corresponden a extractos de trabajos investigativos desarrollados como tesis de distintos niveles, desde pregrado hasta maestría y doctorado, cuya temática gira, en la mayoría de los casos, alrededor de la fuerte influencia que aún ejerce la arquitectura moderna en nuestro contexto contemporáneo. 
El primer grupo de investigaciones aborda el proyecto moderno local, comenzando en la escala urbana, con la pesquisa que realiza Juan Alberto Restrepo sobre la unidad vecinal modelo del plan piloto para Medellín que realizaron los urbanistas Paul Lester Weiner y Josep Lluís Sert en 1950. Mas adelante, tenemos el conjunto industrial de la Compañía Colombiana de Tabacos en Medellín, diseñado por la firma H.M. Rodríguez e hijos en cabeza de Nel Rodríguez, capítulo en el que Oscar Santana aborda la importancia que tienen las nuevas formas de trabajo que aparecen en el siglo xx en los cambios urbanos y arquitectónicos de la época. Posteriormente, está la reflexión que presenta Carlos Mario Pérez sobre la importancia urbana de los pisos bajos en los edificios altos, concentrando su mirada en el concurso para el edificio Avianca en Bogotá, símbolo icónico por excelencia de la modernidad colombiana. Las investigaciones sobre la modernidad en Colombia terminan con el capítulo sobre la fotografía arquitectónica, texto en el que Felipe Villa, Lina María Gonzáles y Leonardo Correa, abren un panorama interesantísimo sobre la manera como cambia la forma de ver la ciudad con la llegada de la modernidad, a través del análisis de la fotografía de arquitectura de mediados del siglo xx.

Seguidamente, se presentan cuatro capítulos que abordan problemas de la arquitectura moderna en diversos lugares del mundo, comenzando con la construcción de la idea de paisaje moderno a partir del análisis de la casa do vidrio de Lina Bo Bardi, en Sao Paulo, Brasil. En ese capítulo, Farhid Maya expone la influencia que tuvo la arquitectura moderna brasileña en el panorama internacional. Luego está el análisis que realiza, desde el punto de vista de la técnica, Juan Fernando Valencia, enfocándose en la definición del concepto de Baukunst con el análisis de la cubierta de la casa Farnsworth de Mies van der Rohe. Por último, en cuanto a los textos enfocados en la arquitectura moderna, están dos capítulos que investigan aquella parte de la arquitectura más cercana a los habitantes: los muebles. John Arango realiza una revisión de los dispositivos mobiliarios de la casa Tugendhat de Mies van der Rohe y propone que las posiciones relativas de estos objetos posibilitan "lugares" en el sentido heideggeriano en el interior de la arquitectura. Por su parte, Viviana Rodríguez hace un análisis del importantísimo papel que jugaron las mujeres arquitectas en el movimiento moderno, particularmente en el diseño del mobiliario, concentrando su atención en la cocina de tres proyectos modernos: la cocina de la Haus am Horn de la 
Bauhaus, la cocina de Frankfurt de Margarette Schutte-Lihotzki y la cocina de la Unidad de Habitación de Marsella de Charlotte Perriand.

Para cerrar el círculo, este libro termina nuevamente en Medellín, pero con una investigación que analiza la manera como esa ciudad moderna, con mas de medio siglo, es apropiada por sus habitantes. Nino Gaviria analiza el fenómeno de "las pintadas" de grafiti y publicitarias como situación estética y social de la Medellín contemporánea.

Estos aportes que se realizan desde la academia a la práctica de la arquitectura, el urbanismo y el diseño están siempre enfocados en entender el papel del proyectista como un intérprete de la cultura y de su propio tiempo, por eso la investigación proyectual es tan rica y tan compleja en sus metodologías y acercamientos. Es esta una mirada de muchas de la manera como se aborda la producción de conocimiento en nuestras disciplinas creativas. 


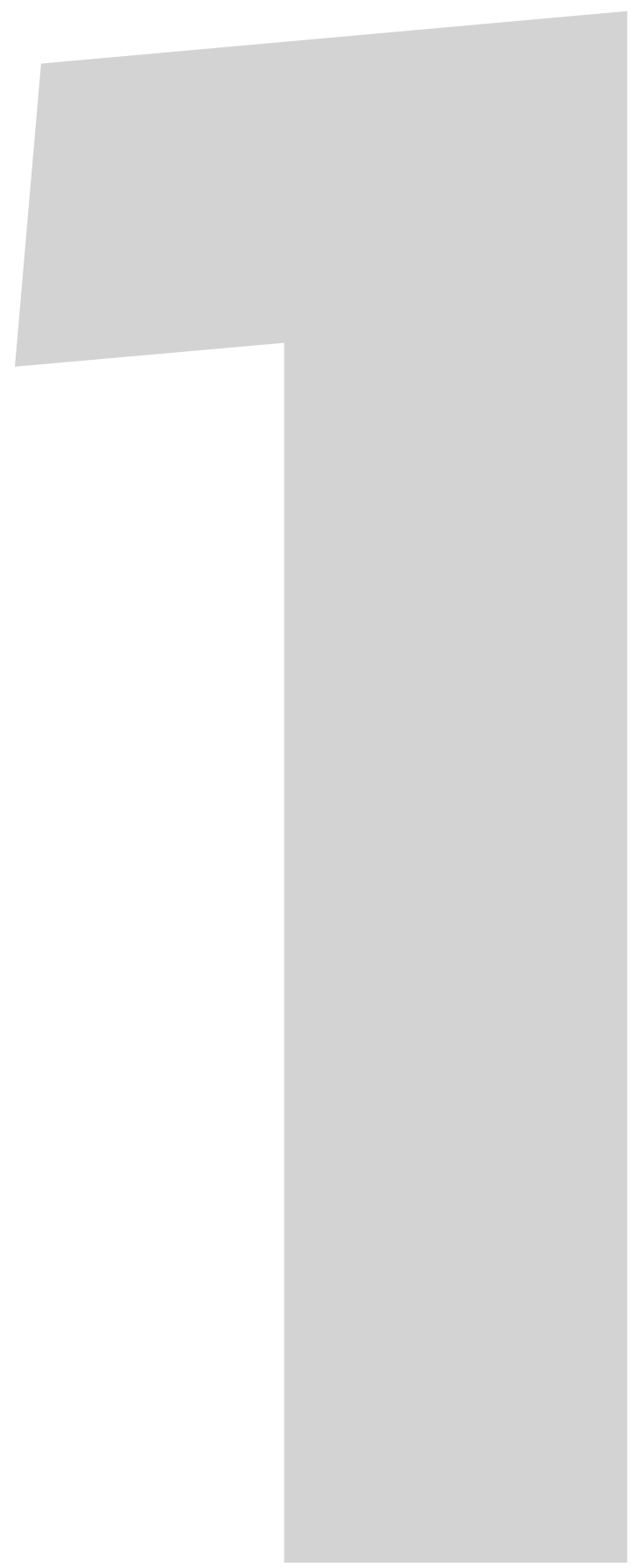




\title{
El complejo panorama de la investigación proyectual en arquitectura y diseño
}

\author{
Natalia Pérez Orrego \\ John Arango Flórez
}

Detallar las características de la investigación proyectual ${ }^{1}$ no es un asunto simple. En primera instancia, las profesiones avocadas a dicho interés como el diseño y la arquitectura tienen una trayectoria muy joven incursionando en el campo investigativo académico; ${ }^{2}$ en segunda instancia, esa inexperiencia ha provocado dilaciones para reconocer las características que componen las relaciones que se pueden tejer entre práctica y teoría. El punto de partida que se les reconoce a las profesiones ya mencionadas es que son actividades

1 En este texto se ha querido ampliar el término anglosajón Design Research hacia otras disciplinas creativas; es por ello que en su reemplazo se propuso la denominación investigación proyectual, con el fin de centrarse en el núcleo que le es común a dichas disciplinas, la proyectación.

2 Vale la pena resaltar que la arquitectura es investigativa desde sus inicios, probando soluciones a partir de la prueba y el error, el libro Architectural Research Methods comienza precisamente con esta idea: "In one sense, architectural research has been conducted thoughtout the history of architecture. The development of particular structural forms or building materials over the centuries is the outcome of trial-and-error experimentation, systematic observation, and application of such building principles to other building projects" (Groat y Wang, 2013, p. 6). 
que centran su práctica en el acto proyectual, lo que significa que siguen un proceso reflexivo y práctico para lanzar una idea. Es por ello que este proceso se ha tomado como parte fundamental, pero no necesariamente definitoria, para esclarecer la investigación proyectual, ya que es usado para capturar, analizar, explorar y transmitir ideas a través de la sensibilidad, la invención, la validación y la implementación.

A continuación, se presentan las particularidades de la práctica proyectual junto con las características que conforman su campo investigativo, para distinguir tres perspectivas investigativas: desde la práctica, sobre la práctica y a través de la práctica, las cuales, si bien parten desde la práctica proyectual, son actividades que derivan en diferentes producciones de conocimiento para el campo disciplinar.

\section{¿Qué es proyectar?}

Como tal sustantivo, el proyecto es el resultado de la acción de proyectar, verbo cuyos significados básicos incluyen idear, trazar, disponer o proponer el plan y los medios para la ejecución de una cosa. Igualmente, proyectar es lanzar hacia adelante o hacia distancia. Por último, proyectar, en su dimensión geométrica, significa trazar líneas rectas desde todos los puntos de un sólido o una figura hacia una superficie plana, el resultado de esta acción es una nueva figura consecuencia del desplazamiento figurado de la originaria (Calderón, 2008, p. 20).

El ejercicio proyectual, como se conoce hoy, nace en la modernidad; hasta el siglo xIx los arquitectos usaban los manuales de estilos y básicamente aplicaban las reglas allí descritas según su necesidad o interés. El ejercicio de la arquitectura entonces se basaba en la composición de piezas prefiguradas en tratados y libros históricos.

En la Bauhaus de Weimar la proyectación comenzó a tomar forma como método de experimentación formal y funcional para solucionar problemas prácticos de la Gesamtkunstwerk 
(obra de arte total), que promulgaban en su manifiesto inicial, ${ }^{3}$ a partir de una mezcla entre el modo de operar del artista moderno, las técnicas industriales y las artesanías premodernas. Esta experimentación pretendía lograr diversas propuestas sin ajustarse a manuales basados en estilos históricos, sino utilizando la propia experiencia del diseñador que, como el artista moderno, plasmaba su subjetividad en la obra, interpretando los aspectos externos que influirían en el resultado final del proyecto.

De esta manera, se podría proponer el diseño de una tetera, una silla, una unidad de viviendas o una fábrica, con las mismas premisas y estrategias formales, pero con resultados propios de la escala y la complejidad técnica. Cada proyecto entonces plantea nuevas maneras de interpretar las situaciones que lo hacen posible, y se desplaza así el concepto de estilo.

Después de la Segunda Guerra Mundial, es en la Hochschule für Gestaltung (HfG) de Ulm en Alemania donde la proyectación toma fuerza desde el punto de vista académico. De esta escuela salen las primeras posturas claras sobre la proyectación como método investigativo con Otl Aicher, Tomás Maldonado y Hans Gugelot inicialmente, y más adelante Bruce Archer, Klaus Krippendorff y Gui Bonsiepe propondrían la proyectación como una manera de crear y evidenciar el conocimiento de las disciplinas propias a sus lógicas procedimentales: la arquitectura y los diseños.

Proyectar es expulsar una nueva conexión, estructura, forma o contenido, en la que se sostiene un proceso de pensamiento analítico y que la mayoría de las veces es disímil o errático, pero siempre con el fin de realizar una proyección hacia el futuro. En suma, y como sostiene Krippendorff (2006), el ejercicio proyectual tiene que ver con lo variable. Así como también, al encarnar el proceso creativo en el artefacto, experiencia o espacio, la proyectación es en sí misma una acción cognitiva (Bigss, 2004; Koskinen et al., 2011; Laurel, 2003).

3 "Wollen, erdenken, erschaffen wir gemeinsam den neuen Bau der Zukunft, der alles in einer Gestalt sein wird: Architektur und Plastik und Malerei". Traducción: "Deseemos, proyectemos, creemos todos juntos la nueva estructura del futuro, en que todo constituirá un solo conjunto, arquitectura, plástica, pintura". Fragmento del manifiesto de la Bauhaus de 1919, Walter Gropius (citado en Droste, 2006, p. 15). 
El proceder proyectual es fundamentalmente un proceder estético, es decir, una construcción que eleva la experiencia a un nivel racional y complejo, y que logra de esta manera reeditar lo existente, lo que genera el advenimiento de nuevos hechos, nuevas formas, nuevos preceptos; en últimas, de conocimiento (Montoya, 2008, p. 87).

\section{Investigación proyectual}

Desde la perspectiva del diseñador, ese carácter prospectivo del proyecto tiende a colisionar con el carácter de repensar que tiene el proceso de investigación. Si se divide el término anglosajón Re-search, ${ }^{4}$ se hace evidente la idea de que investigar es volver sobre acciones o territorios pasados bajo una búsqueda cuidadosa y sistemática de lo que podría decirse que se opone hacia los nuevos territorios a los que se dirige el acto proyectual. Aunque esta diferencia es más que clara, la investigación proyectual se ha acercado a la investigación científica con la intención de darle validez a su proceso investigativo, pero pretender investigar de la misma manera como lo hacen las ciencias traiciona el enfoque al que se dirigen las disciplinas creativas. En términos más precisos, el acto proyectual se ocupa de lo que debería ser, a diferencia de las ciencias exactas que se ocupan por descubrir lo que es. De allí que las disciplinas de la creación no deban tener el mismo tipo de investigación que la ciencia, puesto que las particularidades de la actividad proyectual son opuestas a la actividad científica, se persiguen intenciones distintas. La naturaleza del acto proyectual persigue más bien las singularidades y no las generalidades y regularidades que le interesa distinguir a la ciencia.

Mientras las teorías científicas están basadas solamente en lo que existe y puede ser observado mediante un análisis, el diseño se ocupa de artefactos que todavía no están en uso y no pueden haber sido observados, por lo cual los datos son constitutivamente carentes y las experiencias pueden a lo sumo ser anticipadas. (Krippendorff, 2007, p. 71)

4 Search: Try to find something by looking or otherwise seeking carefully and thoroughly. Research: The systematic investigation into and study of materials and sources in order to establish facts and reach new conclusions. Definiciónes tomadas del diccionario Oxford, en http://www.oxforddictionaries.com/, accedido 26/11/2015 
Como se ha señalado, esas dos áreas disciplinares van en direcciones opuestas, pero paradójicamente la arquitectura y el diseño han recurrido a las humanidades o las ingenierías para que la capacidad predictiva de sus nuevas propuestas tenga una supuesta validez. Esto ha sido contraproducente, porque en el proceso de convertir la investigación proyectual en un problema más científico se desarticulan las significaciones propias de su actividad y se subordina esta a otros saberes que en la mayoría de los casos reconocen en la proyectación una actividad referida solo a la formalización exterior de las cosas.

Al respecto, Faste y Faste (2012) sostienen que cuando la investigación proyectual tiene una orientación empírica, basada en la observación directa del mundo y las personas en su contexto para encontrar las necesidades y las oportunidades de diseño, la investigación corre el riesgo de convertirse en un sinónimo de "investigación etnográfica". Dicho enfoque no es problemático en sí mismo para la investigación proyectual, su problema radica en que se concentra solo en algunos de los múltiples datos que se deben reunir para investigación proyectual. "Un diseñador realmente no quiere convertirse en un antropólogo [...], o en un historiador [...] o en un sociólogo. La investigación en diseño realmente es el diseño del diseño" (Faste, 1995, citado en Faste y Faste, 2012, p. 3).

Este panorama contradictorio deja en claro la necesidad de precisar qué es hacer investigación proyectual. Es por ello que desde los años sesenta se ha teorizado bastante sobre el papel que juega la proyectación en la producción de conocimiento para las disciplinas creativas - arquitectura, diseño industrial, gráfico, de vestuario, entre otras-; pero aún, después de más de 50 años, sigue siendo un campo con vacíos epistemológicas y con una trayectoria muy joven en comparación con la que posee la ciencia; sin embargo, existen puntualidades destacables que comienzan a desentrañar este campo investigativo. Por ejemplo, la autora Nigan Bayazit, en su artículo "Investigating Design: A review of forty years of Design" (2004), propone que lo concerniente a la investigación proyectual es:

- La expresión física de las cosas hechas por el hombre y cómo estas cosas desempeñan su trabajo. 
- Cómo los diseñadores se desempeñan, cómo piensan y cómo llevan a cabo la actividad del diseño.

- Lo que se logra al final de una actividad de diseño específico, cómo aparecen las cosas artificiales y lo que significan.

- La búsqueda sistemática y la adquisición de conocimientos relacionados con la actividad de diseño.

La anterior enumeración de características puede sintetizarse en la definición que realizara Bruce Archer (1981): “[...] la investigación en diseño es una indagación sistemática cuyo objetivo es el conocimiento de, o en, la encarnación de la configuración, composición, estructura, propósito, valor y significado de los sistemas de las cosas realizadas por el hombre" (p. 31).

En estos postulados, que si bien clarifican las cuestiones a las que debe dirigirse la investigación proyectual, se percibe aun una condescendencia con el método científico, al quedarse cortos expresando las características metodológicas solo como sistemáticas y rigurosas, como si de cierta forma la proyectación fuera un método investigativo con características y procesos similares a los convencionales. Revisiones más recientes empiezan a proponer puntos de vista más radicales y menos benévolos con la investigación tradicional, como el de los hermanos Faste (2012), quienes proponen que una de las particularidades más importantes de la investigación proyectual es que su método no es estable. Es decir, cada vez que se investiga se reformula el método porque el acto proyectual en sí es variable, depende de situaciones externas que lo modifican y hacen que el actuar del diseñador cambie con cada proyecto y por lo tanto con cada investigación; esta se diseña cada vez que se aborda, la investigación proyectual es en sí misma una práctica creativa.

A partir de estas particularidades y características, algunos autores han definido varias maneras de operar del diseñador-investigador (Faste y Faste, 2012; Frayling, 1993; Sevalson, 2010), y aunque cada uno plantea una clasificación diferente aquí se destacarán tres que son comunes a todos y que abarcan en gran medida las posibilidades de la investigación proyectual. 
1. Investigación desde la práctica proyectual. En esta el ejercicio proyectual se entiende como investigación. Desde este punto de vista podría decirse que cada vez que se diseña algo se está investigando, ya que los insumos para todo proyecto plantean búsqueda de información (mercado, referentes, usuarios, contextos, técnicas, materiales, etc.), planteamiento de hipótesis (a manera de formalizaciones y espacializaciones, en dibujos, prototipos y demás herramientas de representación) y comprobaciones de diferente índole (técnicas, estéticas, de usabilidad, de confort, etc.), eso sí, con el método variable y errático del acto proyectual. Es un tipo investigación cuyo punto de vista es siempre interno, ya que el documento final, y por lo tanto la producción de conocimiento de esta investigación, es el artefacto mismo.

2. Investigación sobre la práctica proyectual. Este tipo de investigación se enfoca en los procedimientos de la práctica proyectual, por eso el ejercicio más usual es el "análisis crítico de proyectos", porque al entender un proyecto de manera integral se puede dar cuenta de un contexto amplio de conocimiento de la misma práctica proyectual, desde lo específico del ejercicio del proyectista - como los modos de proceder y de pensar-, hasta lo más general —como las visiones de mundo involucradas en el proyecto, su influencia cultural o histórica-. Entonces, con los mismos procesos de pensamiento que se llevan a cabo para dar forma a los artefactos se aporta gran parte del conocimiento del que las disciplinas del diseño disponen. Este tipo de investigación proyectual tiene un punto de vista más bien externo, porque aunque depende profundamente de la misma actividad proyectual para desarrollarse, es la misma actividad proyectual la que se analiza como fenómeno.

Los documentos finales de este tipo de investigación proyectual son usualmente textos analítico-críticos que condensan los resultados de revisiones y reconstrucciones de información textual, gráfica y planimétrica concerniente a los proyectos analizados.

3. Investigación a través de la práctica proyectual. En esta clase de investigación proyectual hay una pregunta general o una cuestión mayor que se trata de resolver 
proyectando algo; entonces el proyecto no es el objetivo final, sino que es parte del proceso investigativo. Problemas como la vivienda colectiva en grandes ciudades o las maneras como se debería acercar a los niños al conocimiento en sus primeros años de formación académica desencadenan ejercicios investigativos que implican a la vez puntos de vista externos (realizando búsqueda de información y análisis desde otras disciplinas u otras maneras de investigar como las ciencias sociales o la medicina) y puntos de vista internos: (proyectando soluciones arquitectónicas de diversa índole - para el caso de la vivienda colectiva - o proponiendo nuevas formas de espacializar las aulas de clase y de formalizar el mobiliario de jardines infantiles contemporáneos). Estos proyectos no necesariamente verán la luz como edificios o artefactos comerciales, pero sin duda aportarán al entendimiento final del problema global investigado. Los documentos finales de este tipo de investigación serán entonces textos analítico-críticos, a la vez que proyectos.

Aunque los dos últimos tipos de investigación proyectual sobre y a través se acercan más a la manera tradicional de investigar y de validar un conocimiento, el reto que se les plantea aquí es cómo este conocimiento regresa y alimenta el primer tipo desde, es decir, cómo es que la práctica proyectual se va a enriquecer de los análisis, críticas y proyectos "ideales" que generan estos dos tipos de investigación. En parte, por eso sería un error dejar de lado el primer tipo, ya que en este subyacen las otras dos, la práctica proyectual en sí es el núcleo de la investigación proyectual.

Para finalizar, vale la pena resaltar que hoy en día es cada vez más aceptada la proyectación como un campo de conocimiento válido; evidencia de esto es que una nueva categoría ha sido anexada a la plataforma $\mathrm{CVLac}^{5}$ denominada Producción en artes, arquitectura y diseño. Aunque los puntajes que se le asignan a esta producción no se equiparan a los

5 CVLAC: "Currículum vitae de Latinoamérica y el Caribe". Es el instrumento o formato electrónico de hoja de vida básico utilizado por los investigadores de América Latina y el Caribe, y es administrado por las instituciones gubernamentales que en cada país de la región controlan y administran la investigación académica, que para el caso específico de Colombia es Colciencias. 
tradicionales artículos científicos, es un avance significativo para la investigación en las disciplinas de la creación en Colombia.

\section{Referencias}

Archer, B. (1981). A View of Nature Design Research. En R. Jacques y J. A. Powell (Eds.), Design: Science: Method (pp. 30-47). Guilford: Westbury House.

Bayazit, N. (2004). Investigating design: A review of forty years of Design. Design Issues, 20(1), 16-29.

Bigss, M. (2004). Editorial: the role of the artefact in art and design research. Working Papers in Art and Design, 3, (1-3).

Calderón, F. (2008). La proyectación: estrategia y proyecto. Recuperado el 24 de 11 de 2013, de eumed.net: http://www.eumed.net/libros-gratis/2008b/411/La\%20Proyectacion\%20 Estrategia\%20y\%20proyecto.htmDroste, M. (2006). Bauhaus. Köln: Taschen.

Faste, R. (1995). Thoughts on design research. Stanford: Rolf A. Faste Foundation For Design Creativity Archives.

Faste, T. y Faste, H. (2012). Desmytifying "design research": design is not research, research is design. IDSA. Education Symposium. Boston, Estados Unidos.

Frayling, C. (1993). Research in art and design. Royal College of Art Research Papers Series, 1(1), 1-5.

Groat, L. y Wang, D. (2013) Architectural research methods: New Jersey: Wiley

Koskinen, I., Zimmerman, J., Binder, T., Redstrom, J. y Wensveen, S. (2011). Design research through practice:from de lab, field, and showroom. Waltham: Morgan Kaufmann.

Krippendorff. (2006). The semantic turn: a new foundation for design. Boca Raton: CRC/Taylor \& Francis.

Krippendorff, K. (2007). Design research, an oximoron? En Design research: Essays and selected projects (pp. 67-80). Zurich: Birkhäuser Verlag.

Laurel, B. (2003). Design research: Methods and perspectives. Boston: The MIT Press.

Montoya, N. (2008). Investigar con el proyecto: la producción de conocimiento desde la disciplina. Proyectiva. Revista de Arquitectura y Proyecto Urbano, 1(1), 84-95.

Sevalson, B. (2010). Discussions and movements in Design Research. FORMakademisk, 3(1), 8-35. 